\title{
A NOTE ON FINITE EULER PRODUCT APPROXIMATIONS OF THE RIEMANN ZETA-FUNCTION
}

\author{
STEVEN M. GONEK
}

(Communicated by Matthew A. Papanikolas)

\begin{abstract}
We construct a family of approximations of the Riemann zetafunction and a closely related function formed from finite Euler products, the pole of the zeta-function, and any zeros the zeta-function might have in the right half of the critical strip. The analysis is unconditional and suggests that if the Riemann Hypothesis is false, then the zeta-function's zeros "arise" in two ways.
\end{abstract}

\section{INTRODUCTION}

In 1 we assumed that the Riemann Hypothesis $(\mathrm{RH})$ holds and investigated the family of functions

$$
\zeta_{X}(s)=P_{X}(s)+\chi(s) P_{X}(\bar{s})
$$

viewing them as models of the Riemann zeta-function, $\zeta(s)$. Here $X \geq 2, s=\sigma+i t$ is a complex variable, $P_{X}(s)$ approximates the Euler product $\prod_{p \leq X^{2}}\left(1-p^{-s}\right)^{-1}$ (see (4) below for the precise definition), and

$$
\chi(s)=\pi^{1 / 2-s} \frac{\Gamma((1-s) / 2)}{\Gamma(s / 2)}
$$

is the factor from the functional equation $\zeta(s)=\chi(s) \zeta(1-s)$. This construction was based on the approximate functional equation for $\zeta(s)$. One advantage to using $P_{X}(s)$ instead of truncations of the Dirichlet series for $\zeta(s)$, as is usually done, is that this forces $\zeta_{X}(s)$ to satisfy a "Riemann hypothesis". (For models built from such Dirichlet polynomials, see Gonek and Montgomery [3].) We showed that $\zeta_{X}(s)$ has the following properties:

(i) Each $\zeta_{X}(s)$ satisfies a Riemann hypothesis in the sense that there exists a positive constant $C_{0}<6.5$ such that every zero in the critical strip with imaginary part greater than $C_{0}$ in absolute value lies on the line $\sigma=\frac{1}{2}$.

(ii) If $\mathrm{RH}$ holds for $\zeta(s)$, then $\zeta_{X}(s)$ is a good approximation of $\zeta(s)$ when $|t|$ and $X$ are sufficiently large and $\sigma$ is greater than, but not too close to, $\frac{1}{2}$.

(iii) If $\mathrm{RH}$ holds for $\zeta(s)$ and $X$ is not too large, then $\zeta_{X}(s)$ has approximately the same number of zeros as $\zeta(s)$ on the critical line, and these are all simple.

Received by the editors April 20, 2013 and, in revised form, October 7, 2013.

2010 Mathematics Subject Classification. Primary 11M06, 11M26.

Key words and phrases. Riemann zeta-function, Euler products, zeros of the zeta-function. Research of the author was supported in part by NSF grant DMS-1200582. 
(iv) If RH holds for $\zeta(s)$, then on intervals between the zeros of $\zeta(s), \zeta_{X}\left(\frac{1}{2}+i t\right)$ tends to $2 \zeta\left(\frac{1}{2}+i t\right)$ as $X$ tends to infinity.

We also observed in [1] that it is more natural to regard $\zeta_{X}(s)$ as an approximation of the function

$$
\mathscr{F}(s)=\zeta(s)+\chi(s) \zeta(\bar{s}) \quad\left(\sigma \geq \frac{1}{2}\right)
$$

rather than of $\zeta(s)$. Our purpose here is to construct functions similar to (11) that approximate $\mathscr{F}(s)$ well in $\sigma \geq \frac{1}{2}$ whether the Riemann Hypothesis is true or not.

It may seem odd to study $\mathscr{F}(s)$ instead of $\zeta(s)$ directly, especially since $\zeta(s)$ is meromorphic and $\mathscr{F}(s)$ is not. However, knowing $\mathscr{F}(s)$ when $\sigma \geq \frac{1}{2}$ is essentially the same as knowing $\zeta(s)$ for $\sigma \geq \frac{1}{2}$ and, therefore, for all $s$ by the functional equation. For example, from $\zeta(s)=\chi(s) \zeta(1-s)$ we see that

$$
\begin{aligned}
\mathscr{F}\left(\frac{1}{2}+i t\right) & =\zeta\left(\frac{1}{2}+i t\right)+\chi\left(\frac{1}{2}+i t\right) \zeta\left(\frac{1}{2}-i t\right) \\
& =2 \zeta\left(\frac{1}{2}+i t\right) .
\end{aligned}
$$

Thus, $\mathscr{F}(s)=2 \zeta(s)$ on the critical line $\sigma=\frac{1}{2}$. When $\sigma \geq \frac{1}{2}$, we have

$$
\mathscr{F}(s)=\zeta(s)\left(1+\chi(s) \frac{\zeta(\bar{s})}{\zeta(s)}\right)
$$

where we define $\zeta(\bar{s}) / \zeta(s)$ at a zero $s=\rho$ or at the pole $s=1$ of $\zeta(s)$ as the limit as $s \rightarrow \rho$ or 1 , respectively. It follows that $|\zeta(\bar{s}) / \zeta(s)|=1$ for all $s$, so if $\mathscr{F}(s)=0$, then either $\zeta(s)=0$ or $|\chi(s)|=1$. Now, we know that if $|\chi(s)|=1$ and $|t|>C_{0}$, the constant in (i) above, then $\sigma=\frac{1}{2}$. Hence, the zeros of $\mathscr{F}(s)$ with $\sigma \geq \frac{1}{2}$ and $|t|>C_{0}$ are either zeros of $\zeta(s)$ or are zeros of the second factor on the right-hand side of (2) and lie on the critical line. However, this factor is identically 2 on $\sigma=\frac{1}{2}$ by the functional equation. It follows that the zeros of $\mathscr{F}(s)$ and $\zeta(s)$ are the same in the region $\sigma \geq \frac{1}{2},|t|>C_{0}$. In particular, if the Riemann Hypothesis holds for $\zeta(s)$, then all the zeros of $\mathscr{F}(s)$ in this region must lie on the critical line. Conversely, if every zero of $\mathscr{F}(s)$ in $\sigma \geq \frac{1}{2},|t|>C_{0}$ is on the critical line, then this is also true for $\zeta(s)$. Since we also know that $\zeta(s)$ has no zeros in $0<|t| \leq C_{0}<6.5$, in fact the "full" Riemann Hypothesis holds for $\zeta(s)$. It is also clear from (2) and the estimate $\chi(s) \ll|s|^{1 / 2-\sigma}$ that $\zeta(s)$ and $\mathscr{F}(s)$ are of the same order in $\sigma \geq \frac{1}{2},|t| \geq 1$. In particular, the truth of the Lindelöf Hypothesis for one function implies it for the other.

For $\frac{1}{2} \leq \sigma<17.5,|t| \leq C_{0}, \mathscr{F}(s)$ has at most finitely many zeros. To see this, we note that the points in the rectangle $[.5,17.5) \times\left[-C_{0}, C_{0}\right]$ for which $|\chi(s)|=1$ form a continuous closed curve $\mathscr{C}$ of finite length which does not pass through $s=1$, plus a point near $s=17.4$. Since $\zeta(s)$ has no zeros in this region, we see from (2) that the only way $\mathscr{F}(s)$ can vanish on $\mathscr{C}$ is if $\arg \chi(s)-2 \arg \zeta(s) \equiv \pi(\bmod 2 \pi)$. That is, if the image of $\mathscr{C}$ under the function $\chi(s) / \zeta(s)^{2}$, which is analytic and nonvanishing in a neighborhood of $\mathscr{C}$, crosses the negative real axis. Clearly this can happen at most finitely many times. There are also zeros near the odd integers $\geq 17$ because on the intervals $(4 n+1,4 n+3), \chi(\sigma)$ tends to $-\infty$ as $\sigma$ approaches the endpoints, and $\chi(\sigma)>-1$ near the center of these intervals when $n \geq 41$

For $T \geq 2$ let $N(T)$ be the number of zeros of $\zeta(s)$ with ordinates in $(0, T]$, let $N^{0}(T)$ be the number of these zeros on the critical line, and let $N^{+}(T)$ be

\footnotetext{
${ }^{1}$ I wish to thank the anonymous referee for pointing this out and for several other helpful suggestions.
} 
the number to the right of it. We also let $N_{\mathscr{F}}(T), N_{\mathscr{F}}^{0}(T)$, and $N_{\mathscr{F}}^{+}(T)$ be the corresponding quantities for $\mathscr{F}(s)$, except that in the case of $N_{\mathscr{F}}(T)$ we only count zeros in our domain of definition, $\sigma \geq \frac{1}{2}, s \neq 1$. If $T$ coincides with the ordinate of one or more zeros, our convention is that $N(T)=\lim _{\epsilon \rightarrow 0+} N(T+\epsilon)$, and similarly for the other counting functions. Clearly

$$
\begin{aligned}
N(T) & =N^{0}(T)+2 N^{+}(T), \\
N_{\mathscr{F}}(T) & =N_{\mathscr{F}}^{0}(T)+N_{\mathscr{F}}^{+}(T) .
\end{aligned}
$$

Moreover, by our previous discussion

$$
\begin{aligned}
& N_{\mathscr{F}}^{0}(T)=N^{0}(T), \\
& N_{\mathscr{F}}^{+}(T)=N^{+}(T)+O(1) .
\end{aligned}
$$

In the next section we shall construct a family of functions $\left\{\mathscr{F}_{X}(s)\right\}$ and state several results about how well they approximate $\mathscr{F}(s)$ and how many zeros they have. We shall give the proofs in Sections 3 and 4.

We conclude this section with two remarks. First, although we shall not use this fact, $\mathscr{F}(s)$ regarded as a function on the whole complex plane satisfies the functional equation

$$
\chi(s) \mathscr{F}(1-s)=\overline{\chi(1-s) \mathscr{F}(s)} .
$$

Secondly, one could just as easily work with the more general functions

$$
\mathscr{F}(s, \theta)=\zeta(s)+e^{i \theta} \chi(s) \zeta(\bar{s}),
$$

where $0 \leq \theta<2 \pi$. Notice, however, that $\mathscr{F}\left(\frac{1}{2}+i t, \pi\right)$ is identically zero.

\section{Approximations of $\mathscr{F}(s)$ And statement of Results}

To construct functions like (1) that approximate $\mathscr{F}(s)$ well even if there are zeros in $\sigma>\frac{1}{2},|t|>C_{0}$, that is, even if the Riemann Hypothesis is false, we use a variant of the hybrid Euler-Hadamard product for the zeta-function of Gonek, Hughes, and Keating [2] that was proved in [1]. Let $X \geq 2$, let $t$ be an arbitrary real number, and set

$$
P_{X}(s)=\exp \left(\sum_{n \leq X^{2}} \frac{\Lambda_{X}(n)}{n^{s} \log n}\right)
$$

where

$$
\Lambda_{X}(n)= \begin{cases}\Lambda(n) & \text { if } n \leq X, \\ \Lambda(n)(2-\log n / \log X) & \text { if } X<n \leq X^{2}, \\ 0 & \text { if } X^{2}<n .\end{cases}
$$

Here $\Lambda(n)$ is von Mangoldt's function, which equals $\log p$ if $n$ is a positive integral power of $p$, and is 0 otherwise. We also set

$$
Z_{X}(s)=\exp \left(\sum_{\rho} F_{2}((s-\rho) \log X)\right) \times \exp \left(-F_{2}((s-1) \log X)\right),
$$

where

$$
F_{2}(z)=2 E_{2}(2 z)-E_{2}(z)
$$

and

$$
E_{2}(z)=\int_{z}^{\infty} \frac{e^{-w}}{w^{2}} d w \quad(z \neq 0)
$$


The hybrid formula asserts that

$$
\zeta(s)=P_{X}(s) Z_{X}(s)\left(1+\mathcal{E}_{X}(t)\right) \quad(\sigma>0),
$$

where

$$
\mathcal{E}_{X}(t) \ll \frac{1}{(|t|+1)^{2} X^{2}}
$$

Next we set

$$
Z_{X}^{+}(s)=\exp \left(\sum_{\beta>1 / 2} F_{2}((s-\rho) \log X)\right) \times \exp \left(-F_{2}((s-1) \log X)\right),
$$

where the sum is over all zeros $\rho=\beta+i \gamma$ of $\zeta(s)$ with $\beta>\frac{1}{2}$. Thus, $Z_{X}^{+}(s)$ is formed from the factors of $Z_{X}(s)$ corresponding to the pole of $\zeta(s)$ at $s=1$ and the zeros of $\zeta(s)$ with real part strictly greater than $\frac{1}{2}$. As our approximations of $\mathscr{F}(s)$ we take the functions

$$
\mathscr{F}_{X}(s)=P_{X}(s) Z_{X}^{+}(s)+\chi(s) P_{X}(\bar{s}) Z_{X}^{+}(\bar{s}),
$$

where $P_{X}(s)$ is given by (44). For $s=\sigma+i t$ with $\sigma \geq \frac{1}{2}$, we also set

$$
\delta(s)=\min _{\beta \leq \frac{1}{2}}|s-\rho| .
$$

Theorem 1. Let $\sigma \geq \frac{1}{2}$ and suppose that $\delta(s)=\min _{\beta \leq 1 / 2}|s-\rho|>0$. Also, let $B>\frac{1}{2}$ and assume that either

$$
X \geq \exp \left(\frac{\log ^{B}(|t|+2)}{\delta(s)}\right)
$$

or

$$
X \geq \exp \left(\frac{2 B \log \log (|t|+3)}{\delta(s)}\right) \quad \text { and } \quad \sigma \geq \frac{1}{2}+\delta(s)
$$

Then

$$
\zeta(s)=P_{X}(s) Z_{X}^{+}(s)\left(1+O\left(\frac{1}{\log ^{2 B-1}(|t|+2)}\right)\right) .
$$

From this we immediately obtain

Corollary 2. Under the same hypotheses as in Theorem 1, we have

$$
\mathscr{F}(s)=\mathscr{F}_{X}(s)\left(1+O\left(\frac{1}{\log ^{2 B-1}(|t|+2)}\right)\right) .
$$

From the proof of Theorem 1 (in particular, see formula (16)) we see that if $\sigma>\frac{1}{2}$ or if $\sigma=\frac{1}{2}$ but $s$ does not coincide with a zero of $\zeta(s)$, then

$$
\lim _{X \rightarrow \infty} P_{X}(s) Z_{X}^{+}(s)=\zeta(s),
$$

and therefore that

$$
\lim _{X \rightarrow \infty} \mathscr{F}_{X}(s)=\mathscr{F}(s) .
$$


Similarly, we easily deduce

Corollary 3. Suppose that $\gamma<\gamma^{\prime}$ are consecutive ordinates of zeros of $\zeta(s)$ on $\sigma=\frac{1}{2}$, that $0<\delta<\frac{1}{2}\left(\gamma^{\prime}-\gamma\right)$, and that $I=\left[\gamma+\delta, \gamma^{\prime}-\delta\right]$. Then

$$
P_{X}\left(\frac{1}{2}+i t\right) Z_{X}^{+}\left(\frac{1}{2}+i t\right) \longrightarrow \zeta\left(\frac{1}{2}+i t\right)
$$

and

uniformly on $I$ as $X \rightarrow \infty$.

$$
\mathscr{F}_{X}\left(\frac{1}{2}+i t\right) \longrightarrow \mathscr{F}\left(\frac{1}{2}+i t\right)
$$

Our final theorem relates the number of zeros of $\mathscr{F}_{X}(s)$ to the number of zeros of $\mathscr{F}(s)$ and therefore of $\zeta(s)$. For $T \geq 2$ let $N_{\mathscr{F}_{X}}^{0}(T)$ be the number of zeros of $\mathscr{F}_{X}(s)$ with ordinates in $(0, T]$ lying on $\sigma=\frac{1}{2}$, and let $N_{\mathscr{F}_{X}}^{+}(T)$ be the number lying strictly to the right of $\sigma=\frac{1}{2}$.

Theorem 4. Suppose that $X, T \geq 2$ and that $\frac{1}{2}+i T$ is not a zero of $\zeta(s)$. Then

$$
N_{\mathscr{F}_{X}}^{+}(T)-N_{\mathscr{F}_{X}}^{+}(7)=N_{\mathscr{F}}^{+}(T)-N_{\mathscr{F}}^{+}(7) .
$$

Alternatively,

$$
N_{\mathscr{F} X}^{+}(T)=N_{\mathscr{F}}^{+}(T)+O_{X}(1) .
$$

If we also have $X \geq \exp \left(\log ^{B} T / \delta\left(\frac{1}{2}+i T\right)\right)$, with $\delta(s)$ as in (9), then

$$
N_{\mathscr{F} X}^{0}(T) \geq N_{\mathscr{F}}^{0}(T)+O(1) \text {. }
$$

The implied constant in the O-term in (12) depends on $X$, but the one in (13) does not. These formulas also hold with $N_{\mathscr{F}}^{+}(T)$ and $N_{\mathscr{F}}^{0}(T)$ replaced by $N^{+}(T)$ and $N^{0}(T)$, respectively.

The last assertion of Theorem 4 follows immediately from the first two and (3).

These results suggest, among other things, that the zeros of the zeta-function on the critical line arise from the functional equation (from which $\mathscr{F}_{X}(s)$ was constructed) in a way that other zeros in the strip (if they exist) do not.

It would be very interesting if one could replace the inequality in (13) by equality, even at the cost of a larger error term.

\section{Proof of Theorem 1}

By (7) and the definition of $Z_{X}^{+}(s)$, we find that

$$
\frac{\zeta(s)}{P_{X}(s) Z_{X}^{+}(s)}=\exp \left(\sum_{\beta \leq 1 / 2} F_{2}((s-\rho) \log X)\right)\left(1+\mathcal{E}_{X}(t)\right) .
$$

To estimate the sum over $\beta$, we observe that integration by parts in (6) leads to

$$
E_{2}(z)=e^{-z} z^{-2}\left(1+O\left(|z|^{-1}\right)\right)
$$

for $|z| \geq 1$. Using this in (5), we find that

$$
F_{2}(z) \ll e^{\max (-\Re z,-\Re 2 z)}|z|^{-2} \quad(|z| \geq 1) .
$$

By assumption $\delta(s) \neq 0$, so we may take $X \geq e^{1 / \delta(s)}$. We then have from (15) that

$$
\sum_{\beta \leq 1 / 2} F_{2}((s-\rho) \log X) \ll \frac{X^{1 / 2-\sigma}}{\log ^{2} X}\left(\sum_{\beta \leq 1 / 2} \frac{1}{(\sigma-1 / 2)^{2}+(t-\gamma)^{2}}\right) .
$$


There are at most $O(\log (|t|+2))$ zeros $\rho$ with $|\gamma-t| \leq 1$, so

$$
\sum_{\beta \leq 1 / 2} \frac{1}{(\sigma-1 / 2)^{2}+(t-\gamma)^{2}} \ll \frac{\log (|t|+2)}{\delta(s)^{2}} .
$$

Hence,

$$
\sum_{\beta \leq 1 / 2} F_{2}((s-\rho) \log X) \ll \frac{X^{1 / 2-\sigma}}{\log ^{2} X} \frac{\log (|t|+2)}{\delta(s)^{2}}
$$

for $\sigma \geq \frac{1}{2}$ and $X \geq e^{1 / \delta(s)}$. If we take $X$ even larger, say

$$
X \geq \exp \left(\frac{\log ^{B}(|t|+2)}{\delta(s)}\right),
$$

the right-hand side of (16) is $O\left(1 / \log ^{2 B-1}(|t|+2)\right)$ for all $\sigma \geq \frac{1}{2}$. On the other hand, if we assume that $\sigma \geq \frac{1}{2}+\delta(s)$ and only take

$$
X \geq \exp \left(2 B \frac{\log \log (|t|+3)}{\delta(s)}\right),
$$

the right-hand side of (16) is

$$
\ll \frac{e^{-2 B \log \log (|t|+3)} \log (|t|+2)}{(2 B \log \log (|t|+3))^{2}} \ll \frac{1}{\log ^{2 B-1}(|t|+2)} .
$$

Combining these estimates with (14) and using (8), we arrive at (10).

\section{Proof of Theorem 4}

We begin by writing

$$
\mathscr{F}_{X}(s)=P_{X}(s) Z_{X}^{+}(s)\left(1+\chi(s) \frac{P_{X}(\bar{s}) Z_{X}^{+}(\bar{s})}{P_{X}(s) Z_{X}^{+}(s)}\right)
$$

and arguing as we did immediately after (2). Now

$$
\left|\frac{P_{X}(\bar{s}) Z_{X}^{+}(\bar{s})}{P_{X}(s) Z_{X}^{+}(s)}\right|=1
$$

for all $s$ with $\sigma \geq \frac{1}{2}$, provided that at a zero or the pole of $Z_{X}^{+}(s)$ we define the quotient to be the limit as $s$ tends to the zero or pole. Then, if $\mathscr{F}_{X}(s)=0$ in the half-plane $\sigma \geq \frac{1}{2}$, either $Z_{X}^{+}(s)=0$ or $|\chi(s)|=1$. If the latter occurs and $|t|>C_{0}$, we must have $\sigma=\frac{1}{2}$. Thus, the only zeros of $\mathscr{F}_{X}(s)$ in $\sigma>\frac{1}{2},|t|>C_{0}$ are those of $Z_{X}^{+}(s)$ or, equivalently, of $\zeta(s)$. But we have seen (see the discussion after (2)) that these are the same as the zeros of $\mathscr{F}(s)$ in $\sigma>\frac{1}{2},|t|>C_{0}$. This proves (11).

By essentially the same argument that we used to estimate the number of zeros of $\mathscr{F}(s)$ in $\frac{1}{2} \leq \sigma<17.5,|t| \leq C_{0}$, we see that $N_{\mathscr{F}_{X}}^{+}(7) \ll_{X} 1$. Hence, for $X, T \geq 2$ we have

which is (12).

$$
N_{\mathscr{F} X}^{+}(T)=N_{\mathscr{F}}^{+}(T)+O_{X}(1)
$$

The zeros of $\mathscr{F}_{X}(s)$ on the critical line $\sigma=\frac{1}{2}$ are the solutions of

$$
\chi\left(\frac{1}{2}+i t\right) \frac{P_{X}\left(\frac{1}{2}-i t\right) Z_{X}^{+}\left(\frac{1}{2}-i t\right)}{P_{X}\left(\frac{1}{2}+i t\right) Z_{X}^{+}\left(\frac{1}{2}+i t\right)}=-1,
$$


and we seek a lower bound for the number of these solutions. Let $f(s)=\arg \chi(s)-$ $2 \arg P_{X}(s) Z_{X}^{+}(s)$, where the arguments are determined by starting with the value 0 at $s=2$ and letting them vary continuously, first along the segment from $s=2$ to $2+i t$, and then horizontally from $2+i t$ to $s=\sigma+i t$. If the horizontal segment passes through a zero or pole of either function, we replace it with the same segment translated up by $\epsilon>0$ and take the limit as $\epsilon \rightarrow 0^{+}$.

Since neither $\chi(s)$ nor $P_{X}(s) Z_{X}^{+}(s)$ vanishes on $\sigma=\frac{1}{2}, f(s)$ is continuous on the vertical segment $\mathscr{L}=\left[\frac{1}{2}, \frac{1}{2}+i T\right]$. Moreover, each time the image of $\mathscr{L}$ under $f(s)$ crosses a horizontal line $y=(2 n+1) \pi$ in the $t y$-plane, we obtain a distinct zero of $\mathscr{F}\left(\frac{1}{2}+i t\right)$. Thus, a lower bound for the number of zeros of $\mathscr{F}\left(\frac{1}{2}+i t\right)$ is

$$
N_{\mathscr{F}_{X}}^{0}(T) \geq \frac{1}{2 \pi}\left|\triangle_{\mathscr{L}}\left(\arg \chi(s)-2 \arg P_{X}(s) Z_{X}^{+}(s)\right)\right|+O(1) .
$$

To estimate $\triangle_{\mathscr{L}} \arg P_{X}(s) Z_{X}^{+}(s)$ here, consider the change in argument around the positively oriented rectangle $\mathcal{R}$ with vertices at $2,2+i T, \frac{1}{2}+i T, \frac{1}{2}$, but with a small semicircle in the lower half-plane replacing a small section of the segment through $s=1$. Then

$$
\triangle \arg _{\mathcal{R}}\left(P_{X}(s) Z_{X}^{+}(s)\right)=2 \pi N^{+}(T)-2 \pi,
$$

where the $-2 \pi$ is from the simple pole of $Z_{X}^{+}(s)$ at $s=1$. By our definition of the argument, the change in $\arg P_{X}(s) Z_{X}^{+}(s)$ along the right and top edges of $\mathcal{R}$ is $\arg P_{X}\left(\frac{1}{2}+i T\right) Z_{X}^{+}\left(\frac{1}{2}+i T\right)$. The change along the left edge is just $-\triangle_{\mathscr{L}} \arg P_{X}(s) Z_{X}^{+}(s)$, and the change along the bottom edge of $\mathcal{R}$ is $-\pi$ since $P_{X}$ and $Z_{X}^{+}$are real on the real axis, apart from the pole of $Z_{X}^{+}$at $s=1$. Thus we find that

$$
\triangle_{\mathscr{L}} \arg P_{X}(s) Z_{X}^{+}(s)=-2 \pi N^{+}(T)+\arg P_{X} Z_{X}^{+}\left(\frac{1}{2}+i T\right)+O(1) .
$$

If we now define $\arg \zeta(s)$ as we $\operatorname{did} \arg P_{X}(s) Z_{X}^{+}(s)$, we see from (10) that

$$
\arg \zeta\left(\frac{1}{2}+i T\right)=\arg P_{X} Z_{X}^{+}\left(\frac{1}{2}+i T\right)+O\left(\frac{1}{\log ^{2 B-1} T}\right)
$$

when $X \geq e^{\log ^{B} T / \delta(1 / 2+i T)}$. Combining this and (18), we obtain

$$
\triangle_{\mathscr{L}} \arg P_{X}(s) Z_{X}^{+}(s)=-2 \pi N^{+}(T)+\arg \zeta\left(\frac{1}{2}+i T\right)+O(1),
$$

provided that $X \geq e^{\log ^{B}(T+2) / \delta(1 / 2+i T)}$.

We also have

$$
\triangle_{\mathscr{L}} \arg \chi(s)=-T \log \frac{T}{2 \pi}+T+O(1)
$$

(see Titchmarsh [4, p. 143]). Using this and (19) in (17), we see that

$$
\begin{aligned}
N_{\mathscr{F}_{X}}^{0}(T) & \left.\geq \frac{1}{2 \pi} \mid T \log \frac{T}{2 \pi}-T-4 \pi N^{+}(T)+2 \arg \zeta\left(\frac{1}{2}+i T\right)\right) \mid+O(1) \\
& \geq \frac{T}{2 \pi} \log \frac{T}{2 \pi}-\frac{T}{2 \pi}-2 N^{+}(T)+\frac{1}{\pi} \arg \zeta\left(\frac{1}{2}+i T\right)+O(1) .
\end{aligned}
$$

By the well-known formula

$$
N(T)=\frac{T}{2 \pi} \log \frac{T}{2 \pi}-\frac{T}{2 \pi}+\frac{1}{\pi} \arg \zeta\left(\frac{1}{2}+i T\right)+O(1),
$$


we now find that

$$
\begin{aligned}
N_{\mathscr{F}_{X}}^{0}(T) & \geq N(T)-2 N^{+}(T)+O(1) \\
& =N^{0}(T)+O(1) .
\end{aligned}
$$

This is the same as

$$
N_{\mathscr{F} X}^{0}(T) \geq N_{\mathscr{F}}^{0}(T)+O(1)
$$

by (3), so we have (13). This completes the proof of Theorem 4 .

\section{REFERENCES}

[1] S. M. Gonek, Finite Euler products and the Riemann hypothesis, Trans. Amer. Math. Soc. 364 (2012), no. 4, 2157-2191, DOI 10.1090/S0002-9947-2011-05546-7. MR2869202

[2] S. M. Gonek, C. P. Hughes, and J. P. Keating, A hybrid Euler-Hadamard product for the Riemann zeta function, Duke Math. J. 136 (2007), no. 3, 507-549. MR2309173(2008e:11100)

[3] Steven M. Gonek and Hugh L. Montgomery, Zeros of a family of approximations of the Riemann zeta-function, Int. Math. Res. Not. IMRN 20 (2013), 4712-4733. MR3118873

[4] E. C. Titchmarsh, The theory of the Riemann zeta-function, 2nd ed., Edited and with a preface by D. R. Heath-Brown. The Clarendon Press, Oxford University Press, New York, 1986. MR $882550(88 \mathrm{c}: 11049)$

Department of Mathematics, University of Rochester, Rochester, New York 14627 E-mail address: gonek@math.rochester.edu 